\title{
Estimation of Nuclear Separation Energy and Its Relation with Q Value
}

\author{
Abhinav Mishra ${ }^{1 *}$, Tanuj Gupta ${ }^{1}$, Bidhubhusan Sahu $^{2}$ \\ ${ }^{1}$ School of Mechanical Engineering, KIIT University, Bhubaneswar, Odisha, India. \\ 2 School of Applied Sciences, KIIT University, Bhubaneswar, India. \\ * Corresponding author. Tel.: +917064168982; email: abhinav.1993mishra@gmail.com \\ Manuscript submitted October 31, 2015; accepted January 4, 2016. \\ doi: 10.17706/ijapm.2016.6.1.17-24
}

\begin{abstract}
The neutron separation energy is the minimum energy required to remove a neutron from a nucleus. It is the difference between the rest mass energy of the nucleus with one less neutron and the actual rest mass energy of the nucleus. Considering Einstein's mass-energy equivalence relation and using the contributions of the liquid-drop model, we first analyze the various terms that build up the Bethe-von Weizsäcker formula. Using this, we estimate the binding energy of a nucleus which in-turn can be used to calculate the neutron separation energies. This was done to obtain the separation energies of different isotopes of Oxygen-8 and Nickel-28. Using the relationship between binding energy and separation energy, the single as well as double neutron separation energies for transition isotopes of Oxygen-8 and Nickel-28 were calculated. We then progressed on to investigate the co-relation of Separation Energies with the Q-Value for a nuclear reaction and discuss its possible consequences for stellar fusion process.
\end{abstract}

Key words: Bethe-von Weizsäcker formula, binding energy, nickel-28, oxygen-8, separation energy.

\section{Introduction}

Protons and neutrons bound tightly together in the nucleus, despite the electrical repulsion of the protons, is an example of strong interaction. This is called the strong nuclear force that acts only at nuclear dimensions. Nuclear structure analysis is more complex than the analysis of many-electron atoms. Therefore, various models were presented to describe the nuclear structure. The two successful but quite different models are:

1) The liquid-drop Model

2) Shell model or Independent Particle Model

In this paper, we focus mainly on the liquid-drop model. First proposed by the Russian Physicist George Gamow in 1928 and then was later built-up by Neils Bohr who suggested that individual nucleons are analogous to the molecules of a liquid drop, held together by short range interactions and surface-tension effects [1]. Section 2 initially discusses the equivalence of mass defect and binding energy and then attempts to build a mathematical analysis for the liquid drop model and the various terms associated with it. We then focus on the estimation of binding energy using theBethe-von Weizsäcker formula [1]. As binding energy is crucial to determining the separation energy, in Section 3 we establish the relationship between the two. Furthermore, we choose the isotopes of 0xygen-8 and Nickel-28 nuclei for determining the separation energies of various transition isotopes. In Section 4 , we relate the $Q$ value of a nuclear reaction with the 
separation energies and discuss its possibilities to learn more about stellar fusion.

\section{Estimation of Binding Energy Using Bethe-von Weizsäcker Formula}

The energy required to pull apart an atomic nucleus into its constituent parts is called the Nuclear Binding Energy. The sum of the masses of distinct nucleons which constitutes a nucleus is usually greater than the mass of that atomic nucleus. This difference in mass is generally termed as Mass Defect. This mass difference is equivalent to the energy required to bind the individual nucleons together despite the proton-proton repulsion to form an atomic nucleus which is a result of Einstein's famous mass-energy equivalence relation given as [1].

$$
E=\Delta m c^{2}
$$

where $\Delta m$ is the mass difference term which can be written in terms of atomic number $(Z)$, neutron number $(N)$ and the respective masses of protons $\left(m_{p}\right)$ and neutrons $\left(m_{n}\right)$ along with the mass of the nucleus ( $m_{\text {nucleus }}$ ) to get a relation for binding energy as follows:

$$
E_{B}=\left(Z m_{p}+N m_{n}-m_{\text {nucleus }}\right) c^{2}
$$

The liquid drop model treats the nucleus as a drop of incompressible nuclear fluid. This model is a rudimentary one and does not explain all the properties of the nucleus, but does explain why most nuclei possesses a spherical shape and helps to estimate the nuclear binding energy [2].

Mathematical analysis of this theory delivers an equation which relates the binding energy of a nucleus in terms of its individual nucleon number i.e, the number of protons and neutrons. This equation contains five terms on its right hand side [2]. Each of the terms in this formula has a theoretical foundation and can be accounted by considering the five types of energy terms given below [2]:

1) Volume Energy Term [1]: Nuclear forces show saturation; only few of the nearest neighbor gets to interact with an individual nucleon. The term of binding energy as $C_{v} A$ is a result of this effect. Here, $C_{V}$ is an experimentally determined constant.

2) Surface Energy Term [1]: As the nucleons on the surface have no neighbors outside them, they are less tightly bound than those in the interior. This leads to a decrease in binding energy and gives a negative energy term proportional to the surface area which is equal to $4 \pi \mathrm{R}^{2}$. This term, $-C_{s} A$ is proportional to $A^{2 / 3}$ as $R$ is proportional to $A^{1 / 3}$.

3) Coulomb Energy Term [1]: The total repulsive electric potential energy is proportional to $Z(Z-1)$ as every single proton repels every other protons. Also, it is inversely proportional to the radius $\mathrm{R}$ and thus to $A^{1 / 3}$.

4) Asymmetry Energy Term [1]: The nucleus must have a balance between the energies associated with the neutrons and the protons to be in a stable, low energy state. This means that for small $A, N$ is close to $Z$ and for larger $A, N$ is greater than $Z$. The best agreement is of the term when it is proportional to $(N-Z)^{2} / A$.

5) Pairing Energy Term [1]: The nuclear force favors pairing of proton and of neutrons. If both $Z$ and $N$ are even, the energy term is positive (more binding) and if both $Z$ and $N$ are odd, the energy term is negative (less binding) and zero otherwise. The best fit to the data occurs as $\pm C_{p} A^{-0.5}$.

Combing the five terms as explained above, we can get a mathematical approximation of the binding energy by the Bethe-von Weizsäcker formula as follows:

$$
E_{B, v W}=C_{v} A-C_{s} A^{2 / 3}-C_{C} \frac{Z\left(Z-C_{Z}\right)}{A^{1 / 3}}-C_{d} \frac{(Z-N)^{2}}{A}-C_{p} \frac{o_{Z}+o_{N}+1}{A^{C_{e}}}
$$


where $C$ represents the constants for the various terms. If the number of protons $(A)$ is odd, $o_{z}$ is 1 whereas it is 0 if $A$ is even. Similarly, for number of neutrons $(N), o_{N}$ follows the same rule. The values of the constants used are as follows [3]:

$$
\begin{aligned}
C_{v} & =15.409 \mathrm{MeV} \\
C_{s} & =16.873 \mathrm{MeV} \\
C_{c} & =0.695 \mathrm{MeV} \\
C_{z} & =1 \mathrm{MeV} \\
C_{d} & =22.435 \mathrm{MeV} \\
C_{p} & =11.155 \mathrm{MeV} \\
C_{e} & =0.5
\end{aligned}
$$

where $\mathrm{MeV}$ is equivalent to the energy an electron picks up in a one million volt electric field i.e $1.602 \mathrm{X}$ $10^{-13}$ Joules.

Using the contributions of Liquid-Drop model and Bethe-von Weizsäcker formula $\left(\mathrm{E}_{\mathrm{B}, \mathrm{vW}}\right)$, we estimate the Binding energy values for various isotopes of 0xygen-8 and Nickel-28 nuclei. Using the binding energy values, we calculate the corresponding separation energies.

\section{Separation Energy}

The minimum energy needed to pull apart a nucleon from a nucleus gives the separation energy. It is how much the rest mass energy of the nucleus is less than that of the nucleus with one less nucleon and the free nucleon [1]. In this paper, we focus on the Neutron Separation Energies.We know by the definition of separation energy for single neutron [4]:

$$
\begin{aligned}
& S_{n}=\left[m\left({ }_{Z}^{\mathrm{A}-1} \mathrm{X}\right)+m_{n}-m\left({ }_{Z}^{A} X\right)\right] c^{2} \\
& =\left[\left\{(A-1-Z) m_{n}+Z m_{p}-\frac{1}{c^{2}} E_{B}(A-1, Z)\right\}+m_{n}\right. \\
& \left.-\left\{(A-Z) m_{n}+Z m_{p^{-}} \frac{1}{c^{2}} E_{B}(A, Z)\right\}\right] c^{2} \\
& S_{n}=E_{B}(A, Z)-E_{B}(A-1, Z)
\end{aligned}
$$

Similarly, two neutron separation energy is given as [4]:

$$
S_{2 n}=E_{B}(A, Z)-E_{B}(A-2, Z)
$$

The equation (12) and (13) given above represents separation energy in terms of binding energies of two consecutive atomic nuclei. Using this relation, we calculated single neutron as well as the double neutron separation energies for different transition isotopes of Nickel-28. 
The calculated values of separation energies for different isotopes were then compared with the experimental values of National Nuclear Data Center, Brookhaven National Laboratory [5]. The results were then plotted as Separation Energy vs. Neutron Number.

\subsection{Choice of Atomic Nucleus}

One of the motives of this paper is to better understand the fusion process in stellar objects specifically under two principal scenarios just before a supernova explosion [6]. When a massive star reaches the final stage in its lifecycle, either it becomes a white dwarf star or it becomes the more common one, a supergiant. Moving our focus to the more common process, a supergiant reaches till the isotope Nickel-56 in its nuclear fusion process just before explosion.

Additionally, the nuclei of Oxygen-8 and Nickel-28 exhibits greater stability as predicted by the Nuclear Shell Model [1].

\subsection{Single Neutron Separation Energies for Different Isotopes of Nickel-28}

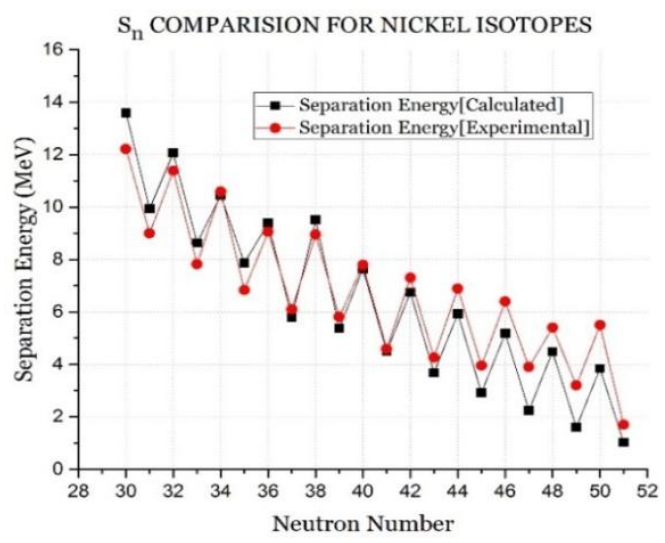

Fig. $1 . \mathrm{S}_{\mathrm{n}}$ values compared with experimental values for consecutive isotopes of $\mathrm{Ni}-28$ showing a saw-tooth pattern.

Table 1. Single Neutron Separation Energies (in MeV) for Various Transition Isotopes of Ni-28

\begin{tabular}{|l|c|c|c|c|c|}
\hline $\begin{array}{c}\text { Transition } \\
\text { Isotopes }\end{array}$ & $\begin{array}{c}\text { Separation } \\
\text { Energy } \\
\text { [Calculated] }\end{array}$ & $\begin{array}{c}\text { Separation Energy } \\
\text { [Experimental] }\end{array}$ & $\begin{array}{c}\text { Transition } \\
\text { Isotopes }\end{array}$ & $\begin{array}{c}\text { Separation } \\
\text { Energy } \\
\text { [Calculated] }\end{array}$ & $\begin{array}{c}\text { Separation Energy } \\
\text { [Experimental] }\end{array}$ \\
\hline${ }_{28}^{58} \mathrm{Ni}$ to ${ }_{28}^{57} \mathrm{Ni}$ & 13.59487 & 12.2163 & ${ }_{28}^{69} \mathrm{Ni}$ to ${ }_{28}^{68} \mathrm{Ni}$ & 4.49188 & 4.586 \\
\hline${ }_{28}^{59} \mathrm{Ni}$ to ${ }_{28}^{58} \mathrm{Ni}$ & 9.94279 & 8.99928 & ${ }_{28}^{70} \mathrm{Ni}$ to ${ }_{28}^{69} \mathrm{Ni}$ & 6.75076 & 7.307 \\
\hline${ }_{28}^{60} \mathrm{Ni}$ to ${ }_{28}^{59} \mathrm{Ni}$ & 12.0651 & 11.38773 & ${ }_{28}^{71} \mathrm{Ni}$ to ${ }_{28}^{70} \mathrm{Ni}$ & 3.67532 & 4.264 \\
\hline${ }_{28}^{61} \mathrm{Ni}$ to ${ }_{28}^{60} \mathrm{Ni}$ & 8.62893 & 7.82011 & ${ }_{28}^{72} \mathrm{Ni}$ to ${ }_{28}^{71} \mathrm{Ni}$ & 5.93156 & 6.891 \\
\hline${ }_{28}^{62} \mathrm{Ni}$ to ${ }_{28}^{61} \mathrm{Ni}$ & 10.43819 & 10.5959 & ${ }_{28}^{73} \mathrm{Ni}$ to ${ }_{28}^{72} \mathrm{Ni}$ & 2.92692 & 3.953 \\
\hline${ }_{28}^{63} \mathrm{Ni}$ to ${ }_{28}^{62} \mathrm{Ni}$ & 7.86839 & 6.83728 & ${ }_{28}^{74} \mathrm{Ni}$ to ${ }_{28}^{73} \mathrm{Ni}$ & 5.17832 & 6.4 \\
\hline${ }_{28}^{64} \mathrm{Ni}$ to ${ }_{28}^{63} \mathrm{Ni}$ & 9.37925 & 9.05747 & ${ }_{28}^{75} \mathrm{Ni}$ to ${ }_{28}^{74} \mathrm{Ni}$ & 2.23944 & 3.9 \\
\hline${ }_{28}^{65} \mathrm{Ni}$ to ${ }_{28}^{64} \mathrm{Ni}$ & 5.78721 & 6.09808 & ${ }_{28}^{76} \mathrm{Ni}$ to ${ }_{28}^{75} \mathrm{Ni}$ & 4.48416 & 5.4 \\
\hline${ }_{28}^{66} \mathrm{Ni}$ to ${ }_{28}^{65} \mathrm{Ni}$ & 9.50747 & 8.9524 & ${ }_{28}^{77} \mathrm{Ni}$ to ${ }_{28}^{76} \mathrm{Ni}$ & 1.60652 & 3.2 \\
\hline${ }_{28}^{67} \mathrm{Ni}$ to ${ }_{28}^{66} \mathrm{Ni}$ & 5.38499 & 5.8083 & ${ }_{28}^{78} \mathrm{Ni}$ to ${ }_{28}^{77} \mathrm{Ni}$ & 3.84306 & 5.5 \\
\hline${ }_{28}^{68} \mathrm{Ni}$ to ${ }_{28}^{67} \mathrm{Ni}$ & 7.64384 & 7.792 & ${ }_{28}^{79} \mathrm{Ni}$ to ${ }_{28}^{78} \mathrm{Ni}$ & 1.02265 & 1.7 \\
\hline
\end{tabular}

For single neutron separation energy, the calculated values produce the same saw-tooth curve as 
predicted by the Weizsäcker formula and substantiated by the experimental data (see Fig. 1). According to the last pairing term in the formula, an even number of particles is more stable than odd number (see Table 1). The tendency to form proton pairs and neutron pairs is a consequence which arises from this energy term [1].

\subsection{Two Neutron Separation Energies for Different Isotopes of Nickel-28}

For two neutron separation energy, we notice a steady decline in separation energy with increase in neutron number (see Table 2). This is equivalent to joining the crests of the curve which can be seen in Fig. 2.

Table 2. Two Neutron Separation Energies (in MeV) for Various Transition Isotopes of Ni-28

\begin{tabular}{|c|c|c|c|c|c|}
\hline $\begin{array}{c}\text { Transition } \\
\text { Isotopes }\end{array}$ & $\begin{array}{c}\text { Separation } \\
\text { Energy } \\
\text { [Calculated] }\end{array}$ & $\begin{array}{c}\text { Separation Energy } \\
\text { [Experimental] }\end{array}$ & $\begin{array}{c}\text { Transition } \\
\text { Isotopes }\end{array}$ & $\begin{array}{c}\text { Separation } \\
\text { Energy } \\
\text { [Calculated] }\end{array}$ & $\begin{array}{c}\text { Separation Energy } \\
\text { [Experimental] }\end{array}$ \\
\hline${ }_{28}^{58} \mathrm{Ni}$ to ${ }_{28}^{56} \mathrm{Ni}$ & 23.53129 & 22.4639 & ${ }_{28}^{69} \mathrm{Ni}$ to ${ }_{28}^{67} \mathrm{Ni}$ & 12.13572 & 12.379 \\
\hline${ }_{28}^{59} \mathrm{Ni}$ to ${ }_{28}^{57} \mathrm{Ni}$ & 23.53767 & 21.2155 & ${ }_{28}^{70} \mathrm{Ni}$ to ${ }_{28}^{68} \mathrm{Ni}$ & 11.24264 & 11.893 \\
\hline${ }_{28}^{60} \mathrm{Ni}$ to ${ }_{28}^{58} \mathrm{Ni}$ & 23.56889 & 20.38701 & ${ }_{28}^{71} \mathrm{Ni}$ to ${ }_{28}^{69} \mathrm{Ni}$ & 10.42608 & 11.57 \\
\hline${ }_{28}^{61} \mathrm{Ni}$ to ${ }_{28}^{59} \mathrm{Ni}$ & 20.79031 & 19.20784 & ${ }_{28}^{72} \mathrm{Ni}$ to ${ }_{28}^{70} \mathrm{Ni}$ & 9.60688 & 11.155 \\
\hline${ }_{28}^{62} \mathrm{Ni}$ to ${ }_{28}^{60} \mathrm{Ni}$ & 19.06713 & 18.20784 & ${ }_{28}^{73} \mathrm{Ni}$ to ${ }_{28}^{71} \mathrm{Ni}$ & 8.85849 & 10.845 \\
\hline${ }_{28}^{63} \mathrm{Ni}$ to ${ }_{28}^{61} \mathrm{Ni}$ & 18.30659 & 17.4336 & ${ }_{28}^{74} \mathrm{Ni}$ to ${ }_{28}^{72} \mathrm{Ni}$ & 8.10525 & 10.4 \\
\hline${ }_{28}^{64} \mathrm{Ni}$ to ${ }_{28}^{62} \mathrm{Ni}$ & 17.24765 & 16.49525 & ${ }_{28}^{75} \mathrm{Ni}$ to ${ }_{28}^{73} \mathrm{Ni}$ & 7.41776 & 10.3 \\
\hline${ }_{28}^{65} \mathrm{Ni}$ to ${ }_{28}^{63} \mathrm{Ni}$ & 15.16646 & 15.75555 & ${ }_{28}^{76} \mathrm{Ni}$ to ${ }_{28}^{74} \mathrm{Ni}$ & 6.72359 & 9.3 \\
\hline${ }_{28}^{66} \mathrm{Ni}$ to ${ }_{28}^{64} \mathrm{Ni}$ & 15.29468 & 15.0504 & ${ }_{28}^{77} \mathrm{Ni}$ to ${ }_{28}^{75} \mathrm{Ni}$ & 6.09068 & 8.6 \\
\hline${ }_{28}^{67} \mathrm{Ni}$ to ${ }_{28}^{65} \mathrm{Ni}$ & 14.89246 & 14.76 & ${ }_{28}^{78} \mathrm{Ni}$ to ${ }_{28}^{76} \mathrm{Ni}$ & 5.44959 & 8.7 \\
\hline${ }_{28}^{68} \mathrm{Ni}$ to ${ }_{28}^{66} \mathrm{Ni}$ & 13.02883 & 13.6 & ${ }_{28}^{79} \mathrm{Ni}$ to ${ }_{28}^{77} \mathrm{Ni}$ & 4.86572 & 7.1 \\
\hline
\end{tabular}

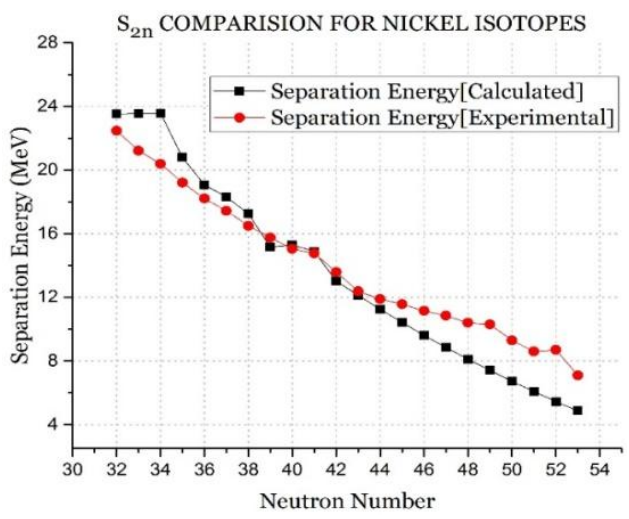

Fig. 2. $\mathrm{S}_{2 \mathrm{n}}$ values compared with experimental values for consecutive isotopes of $\mathrm{Ni}-28$ showing approximately a linear curve.

\subsection{Single Neutron Separation Energies for Different Isotopes of Oxygen-8}

Looking at Table 3, we can infer that separation energy is noticeably higher if the initial number of neutrons is even [7]. If a neutron is removed from a nucleus with an even number of neutrons, a pair must be broken up that requires additional energy [7] as is seen in Fig. 3. 
Table 3. Single Neutron Separation Energies (in MeV) for Various Transition Isotopes of 0-8

\begin{tabular}{|c|c|c|}
\hline Transition Isotopes & Separation Energy [Calculated] & $\begin{array}{c}\text { Separation Energy } \\
\text { [Experimental] }\end{array}$ \\
\hline${ }_{8}^{16} \mathrm{O}$ to ${ }_{8}^{15} \mathrm{O}$ & 15.5173 & 15.6639 \\
\hline${ }_{8}^{17} \mathrm{O}$ to ${ }_{8}^{16} \mathrm{O}$ & 7.1907 & 4.1431 \\
\hline${ }_{8}^{18} \mathrm{O}$ to ${ }_{8}^{17} \mathrm{O}$ & 10.3279 & 8.0454 \\
\hline${ }_{8}^{19} \mathrm{O}$ to ${ }_{8}^{18} \mathrm{O}$ & 3.1473 & 3.9560 \\
\hline${ }_{8}^{20} \mathrm{O}$ to ${ }_{8}^{19} \mathrm{O}$ & 5.7057 & 7.6080 \\
\hline${ }_{8}^{21} \mathrm{O}$ to ${ }_{8}^{20} \mathrm{O}$ & 1.2200 & 3.8060 \\
\hline${ }_{8}^{22} \mathrm{O}$ to ${ }_{8}^{21} \mathrm{O}$ & 3.9553 & 6.8500 \\
\hline
\end{tabular}

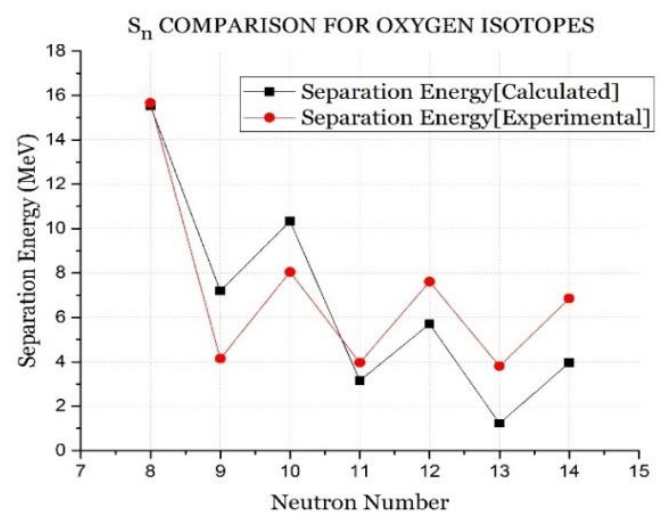

Fig. 3. $S_{n}$ values compared with experimental values for consecutive isotopes of $0-8$ showing a saw-tooth pattern.

\subsection{Two Neutron Separation Energies for Different Isotopes of Oxygen-8}

Table 4. Two Neutron Separation Energies (in MeV) for Various Transition Isotopes of 0-8.

\begin{tabular}{|c|c|c|}
\hline Transition Isotopes & Separation Energy [Calculated] & $\begin{array}{c}\text { Separation Energy } \\
\text { [Experimental] }\end{array}$ \\
\hline${ }_{8}^{16}$ O to ${ }_{8}^{14} \mathrm{O}$ & 28.6132 & 28.8871 \\
\hline${ }_{8}^{17} \mathrm{O}$ to ${ }_{8}^{15} \mathrm{O}$ & 22.7081 & 19.8070 \\
\hline${ }_{8}^{18}$ O to ${ }_{8}^{16} \mathrm{O}$ & 17.5186 & 12.1884 \\
\hline${ }_{8}^{19} \mathrm{O}$ to ${ }_{8}^{17} \mathrm{O}$ & 13.4752 & 12.0010 \\
\hline${ }_{8}^{20}$ O to ${ }_{8}^{18} \mathrm{O}$ & 8.8529 & 11.5637 \\
\hline${ }_{8}^{21}$ O to ${ }_{8}^{19} \mathrm{O}$ & 6.9257 & 11.4140 \\
\hline
\end{tabular}

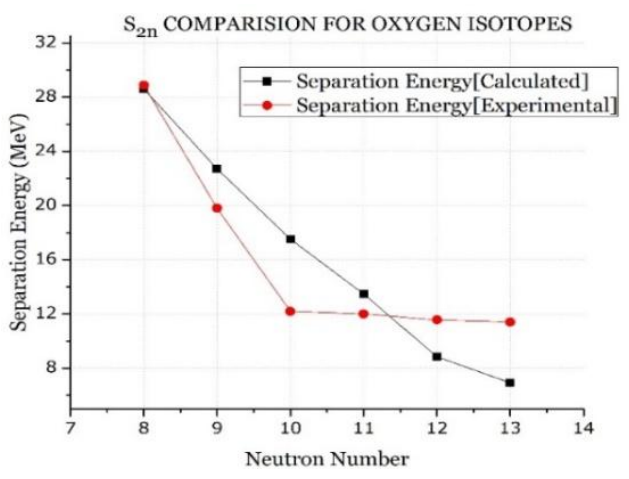

Fig. 4. $S_{2 n}$ values compared with experimental values for consecutive isotopes of 0-8. 
As we can see in Fig.4 that the calculated separation energy varies more from the experimental data (see Table 4). This is because the calculated data is obtained from the Bethe-von Weizsäcker formula which is based on the contributions of the liquid drop model that is more suitable for heavier nuclei.

\section{Separation Energy and Q-Value}

The amount of energy released by a nuclear reaction is given by its $Q$ value. It depends on the masses of reactants and products. $Q$ value for a nuclear reaction can be positive or negative. When it is positive, the reaction is an exothermic reaction whereas when it is negative, the reaction is endothermic [8]. Let us consider a spontaneous nuclear reaction represented by [8]:

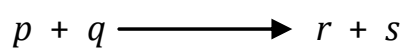

where $p$ and $q$ are the reactants and $\mathrm{r}$ and $\mathrm{s}$ are the products. The mass energy balance involving the kinetic energies and the rest mass energies can be expressed as [9]:

$$
\left(K E_{p}+m_{p} c^{2}\right)+\left(K E_{q}+m_{q} c^{2}\right) \longrightarrow\left(K E_{r}+m_{r} c^{2}\right)+\left(K E_{s}+m_{s} c^{2}\right)
$$

The difference between kinetic energies of products and reactants is equivalent to the difference between the mass of reactants and products [9]. Therefore, the $Q$ value for this reaction is given as:

$$
\begin{aligned}
Q & =\left(m_{p}+m_{q}\right) c^{2}-\left(m_{r}+m_{s}\right) c^{2} \\
& =\left(E_{B} r+E_{B} s\right)-\left(E_{B} p+E_{B} q\right)
\end{aligned}
$$

where $m_{x}$ represents the masses of the individual nuclei and $E_{B}$ represents its binding energies. $Q$ values for the removal of one or two neutrons are defined as:

$$
\begin{gathered}
S_{n}=-Q_{n}=E_{B}(A, Z)-E_{B}(A-1, Z) \\
S_{2 n}=-Q_{2 n}=E_{B}(A, Z)-E_{B}(A-2, Z)
\end{gathered}
$$

Knowing whether the $\mathrm{Q}$ value for a nuclear reaction is positive or negative, we can determine its feasibility. In the case of fusion reactions occurring in more common supergiant stars, the lighter elements fuse together to form heavier elements up to Nickel-56. This isotope undergoes radioactive decay into Iron-56 which is the most stable nuclei as it has the highest binding energy per nucleon. It is also the last element that exothermically produces a net release of energy i.e. it has a positive $Q$ value [10].In case of a neutron rich environment, neutrons maybe added to Nickel-56 to form its heavier isotopes instead of decaying into Iron-56 or on the other hand, these neutrons may first decay into protons which with enough energy may fuse with Nickel-56 to form heavier elements.

\section{Acknowledgment}

The registration of this paper is supported by KIIT University, Bhubaneswar, India.

\section{References}

[1] Krane, K. S. (1988). Introductory Nuclear Physics. New Jersey: Wiley. 
[2] Ernest, M, \& Henley, A. G. (2006). Subatomic Physics Book. Singapore: World Scientific Publishing Co. Ltd.

[3] Chowdhury, P. R., \& Basu, D. N. (2006). Nuclear matter properties with the re-evaluated coefficients of liquid drop model. Acta Physica Polonica B, 37, 1833-1846.

[4] Brink, D. M. (2005), Nuclear Superfluidity: Pairing in Finite Systems. Cambridge: Cambridge University Press.

[5] National Nuclear Data Center, Brookhaven National Laboratory. Based on ENSDF and the Nuclear Wallet Cards. Retrieved, from the website: www.nndc.bnl.gov/chart

[6] François, P., et al. (2004). The evolution of the Milky Way from its earliest phases: Constraints on stellar nucleosynthesis. Astronomy and Astrophysics, 421(2), 613-621.

[7] Dommelen, L. V. (2013). Quantum Mechanics for Engineers. Retrieved, from the website: www.eng.fsu.edu/dommelen

[8] Nuclear Masses and Binding Energies, Information and Communication Technology. Retrieved, from the website: http://pms.iitk.ernet.in/wiki/index.php/Nuclear_Masses_and_Binding_Energies

[9] Ragheb, M. (2014). Nuclear processes, the strong force. Nuclear and Renewable Energy. Illinois: University of Illinois.

[10] Burbidge, E. M. et al. (1957). Synthesis of elements in stars. Rev. Mod. Phys., 29, 547-650.

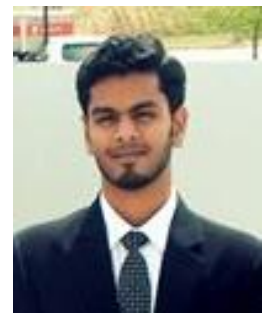

Abhinav Mishra was born in Delhi, the capital city of India on April 17, 1993. He is currently a final year student pursuing the B.Techin mechanical (automobile) engineering from Kalinga Institute of Industrial Technology, KIIT University, Bhubaneshwar, Odisha, India.

His research interest lies in theoretical astrophysics, galactic structure and evolution, neutron stars, dark matter and dark energy, and nuclear astrophysics. Mr. Mishra is a member of the American Physical Society (APS) and Society of Automotive Engineers (SAE).

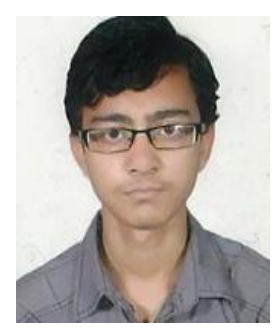

Tanuj Gupta was born in Saptari district of Nepal on Jan 6, 1995. He is currently a final year student pursuing the B.Tech in mechanical engineering from Kalinga Institute of Industrial Technology, KIIT University, Bhubaneshwar, Odisha, India.

His topics of research interests are nuclear astrophysics, stellar nucleosynthesis, neutron Stars and high energy astrophysics and cosmology. Mr. Gupta is a member of the American Physical Society (APS).

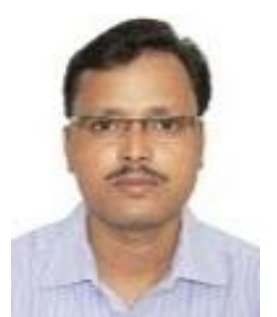

BidhubhusanSahuwas born in Angul district of Odisha, India on May 3, 1980. He finished his B.Sc. degree in physics honours from Talcher College, Talcher, Odisha, India in 2000, a M.Sc. degree in physics and the M.Phil degree in physics from Utkal University, Bhubaneswar, India in 2002 and 2005 respectively. He then completed his Ph.D. degree in physics from North Orissa University, Baripada, India in 2011.

Presently, he is working as an assistant professor in the School of Applied Sciences, KIIT University, bhubaneswar, India. He has published several papers in various international/national Journals. His current research interest lies in the study of scattering and fusion phenomena in heavy-ion collisions. 